\title{
Limbal Disease in Trachoma and Other Ocular Chlamydial Infections: Risk Factors for Corneal Vascularisation
}

\author{
C. R. DAWSON, R. JUSTER, R. MARX, M. T. DAGHFOUS, and A. BEN DJERAD \\ California and Tunis
}

\begin{abstract}
Summary
Ocular infection with Chlamydia trachomatis, whether of genital or endemic trachoma origin, usually produces diffuse infiltration and swelling of the scleral limbus, grey infiltrates of the corneal limbus, and superficial extension of vessels onto the corneal limbus. In genitally transmitted $C$. trachomatis infections, subepithelial infiltrates have been reported as well. In classic endemic trachoma, limbal changes also include limbal follicles which resolve, leaving Herbert's peripheral pits, and an extensive vascular pannus.

To evaluate the limbal changes in trachoma, follow-up studies were done in 19861987 in a group of 213 children originally seen between 1969-1972. Pannus formation occurred at a much earlier age than conjunctival scar formation and was an excellent predictor of later severe conjunctival scarring. The evidence from this study suggests that the mechanisms for corneal pannus formation from the limbus are quite different from those for scarring of the conjunctiva.
\end{abstract}

Following the first isolation of Chlamydia trachomatis in the 1950s, the intense interest in differentiating genitally transmitted $C$. trachomatis eye infection and classic trachoma led to a careful but often conflicting series of clinical descriptions of these two chlamydial syndromes. ${ }^{1,2,3}$ In order to delineate the natural history of limbal disease in endemic trachoma, we report here the relationship and sequence of limbal changes in a cohort of patients in Tunisia followed over 16 to 18 years.
The chlamydia are a unique group of small bacteria comprising a single order and a single family, and one genus, the Chlamydia, with two species: Chlamydia trachomatis and Chlamydia psittaci. ${ }^{4}$ The $C$. trachomatis agents are for the most part human pathogens. The agents of trachoma (usually associated with serotypes $\mathrm{A}, \mathrm{B}, \mathrm{Ba}$ and $\mathrm{C}$ ) and the genitally transmitted agents (serotypes $\mathrm{D}$ through $\mathrm{K}$ ) constitute a sub-group of $C$. trachomatis. This sub-group of agents infects the epithelium of mucosal surfaces, particularly

From: Francis I. Proctor Foundation for Research in Ophthalmology and Scientific Computing Center of the University of California, San Francisco and L'Institut d'Ophtalmologie de Tunis.

Supported in part by grants from The Edna McConnell Clark Foundation, the US National Institutes of Health, National Eye Institute EY.00427, and from the Department of Health and Human Services, Office of International Health PL.480 03-024.

Studies were carried out under Approval No. 460590-10 of the University of California, San Francisco, Committee on Human Research.

Correspondence to: Chandler R. Dawson, MD, Francis I. Proctor Foundation for Research in Ophthalmology, University of California, San Francisco, San Francisco, CA 94143-0412, USA. 
the genital tract and eye. The trachoma-inclusion conjunctivitis group of organisms are very widely distributed: Blinding trachoma affects 600 million people and genital chlamydialinfections are highly endemic in all human populations. All members of the trachoma-inclusion conjunctivitis group of organisms are capable of infecting the conjunctiva and of causing various degrees of corneal involvement.

\section{Corneal Disease in Chlamydial Infections}

The changes at the limbus in ocular chlamydial infections include:

- Limbal oedema and infiltration

- Greyish limbal infiltrates

- Superficial extension of vessels

In the genitally transmitted Chlamydia trachomatis infections that involve the eye, subepithelial infiltrates and chronic epithelial keratitis have been reported by many observers. ${ }^{5,6,7}$

In classic endemic trachoma the limbal changes also include:

- Limbal follicles, which destroy the connective tissue in the scleral overhang, leaving behind scarred depressions called Herbert's peripheral pits, a pathognomonic sign unique to trachoma.

- The vascular extension in trachoma consists of a fibrovascular membrane beyond the limit of the scleral opacity. The extension of vessels usually is an even advance of neovascularisation, usually twice as extensive at the upper limbus as compared to the lower.

-Pannus ulcers are shallow defects of the epithelium and superficial stroma that form immediately in advance of the vessels.

-Epithelial keratitis on the upper cornea is also described as a characteristic finding in trachoma.

\section{Experimental Design}

Between 1969 and 1972, our group carried out a longitudinal study of the epidemiology and natural history of trachoma in a sub-Saharan village in the south of Tunisia. ${ }^{8}$ All persons (about 2,000) in this village were examined for trachoma. In this village all children had acquired active trachoma by the age of two years. Over the three year period of the initial study there was no change in the prevalence of the disease.

By the early 1980s the prevalence of active trachoma declined in this village, where systematic antibiotic treatment was being carried out and where there was marked economic development.

For the follow-up study in 1986-1987, 300 persons were selected at random for follow-up examination from a computerised list of persons who had been examined from 1969 to 1972. These individuals were between the ages of one and 28 years at the time of their initial examination. A total of 213 persons were identified and examined at follow-up. The ages at the follow-up examination ranged from 16 to 32 years.

\section{Methods}

Examination technique and clinical scoring: All examinations at the initial survey 19691972 and the follow-up surveys 1986-1987 were carried out with standard model 900 Haag-Streit slitlamps. The signs recorded at each examination included:

Trichiasis/entropion (T/E)

Conjunctival follicles $(\mathrm{F})$

Maturity of follicles (first examination only) (FM)

Diffuse infiltration and papillary hypertrophy $(\mathrm{P})$

Conjunctival scarring (C)

Corneal vascularisation (pannus) (V)

Corneal infiltration (first examination only) (I)

Conjunctivitis (Co)

The two signs upon which this report is based were recorded using the following scales recommended by WHO: ${ }^{9}$

The scoring for all signs was based on the recommendation of the World Health Organisation. ${ }^{9}$ The scoring for the conjunctival scarring and vascular pannus was as follows:

Conjunctival scarring (C)

$\mathrm{C}_{0}$ No scarring on the conjunctiva

$\mathrm{C}_{1}$ Mild: Fine scattered scars on the upper tarsal conjunctiva, or 
scars on other parts of the conjunctiva

$\mathrm{C}_{2}$ Moderate: More severe scarring but without shortening or distortion of the upper tarsus

$\mathrm{C}_{3}$ Severe: Scarring with distortion of the upper tarsus

Vascular pannus (V)

Vessels (measured from the upper limbus)

$\mathrm{V}_{1}$ 0.5-0.9+ $\mathrm{mm}$ extension beyond normal limbal opacity, as demonstrated by direct focal illumination (micropannus)

$\mathrm{V}_{2}$ 1.0-1.9+ mm extension

$V_{3}$ 2.0-3.9+ mm extension

$\mathrm{V}_{4}$ 4.0-5.9+ $\mathrm{mm}$ extension

$\mathrm{V}_{5} 6.0 \mathrm{~mm}$ or more extension

Clinical observations were reported on a standard form. The examiners were never aware of previous clinical findings. The information from the forms was transferred for computer processing to either magnetic tapes or discs. Data analysis was then carried out in San Francisco using standard statistical software packages. The records stored on magnetic media were verified using the original data collection sheet.

\section{Results}

The results of this survey (reported previously) from 1969-1972 indicated that trachoma was highly endemic in this village with all children having acquired signs of infection by the age of two years. ${ }^{8}$ The active inflammatory disease appeared to have disappeared by the time most children were adolescents. The age distribution of conjunctival scarring showed that only two of the 57 children four years and under had any conjunctival scarring, but conjunctival scarring in those five and older became progressively more marked with increasing age (Fig. 1).

At the follow-up examinations in 1986 and 1987, conjunctival scarring was present in nearly the entire population examined (Fig. 2). Severe scarring, however, involved 20 per cent of those four and under at the original examination, about 40 per cent of those who were originally five to 19 at the initial examination and about 70 per cent of those who had been 20 to 30 years old (Fig. 2).

In contrast to conjunctival scarring, corneal vascularisation was present in 85 per cent of the 57 children under the age of four years (Fig. 3). Even for those children examined during the first year of life, three out of six had evidence of corneal vascularisation already; vascularisation was progressively more severe

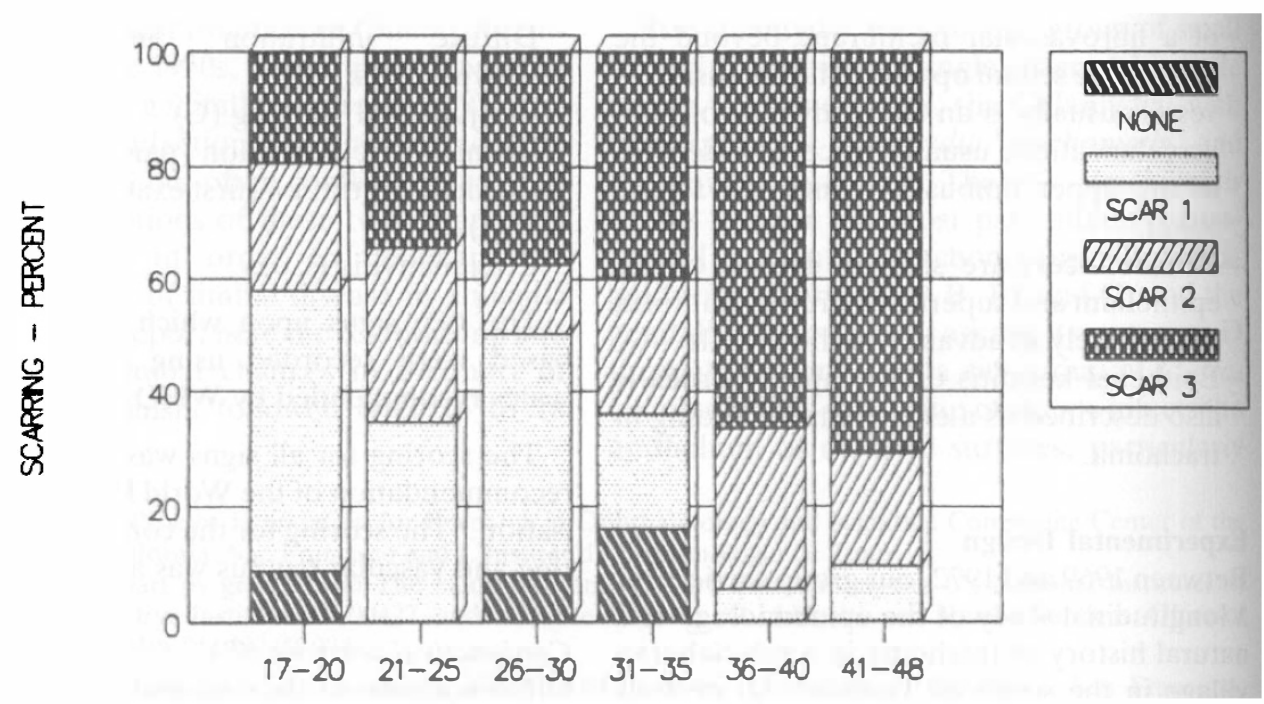

AGE AT SECOND EXAMINATION 1986-87

Fig. 1. Conjunctival scars in trachoma. 16 year follow up exams 1986-1987. 


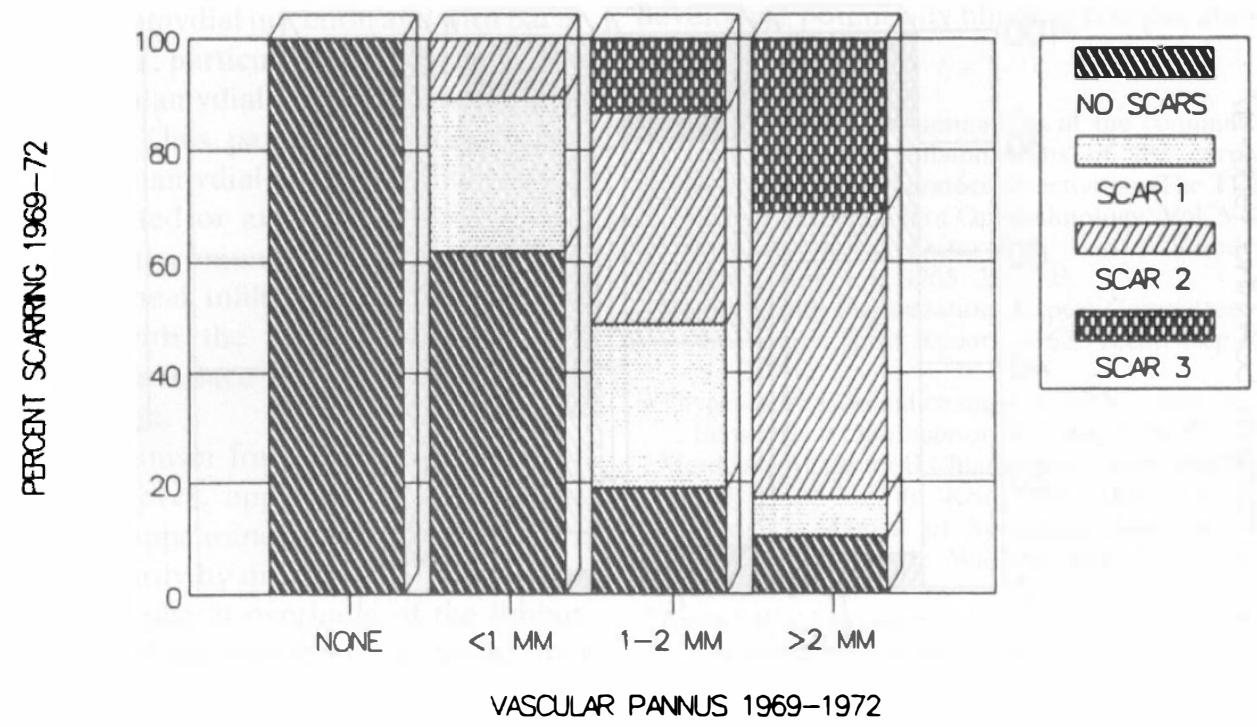

Fig. 2. Trachoma in Tunisia 1969-1972. Pannus and conjunctival scarring.

with advancing age (Fig. 3). It is apparent from a comparison of Figs. 1 and 3 that corneal vascularisation occurred much more readily than detectable conjunctival scarring in these children with trachoma.

For the initial examination in 1969-1972, the degree of corneal pannus and of conjunctival scarring was compared directly (Fig. 4). Most of the children with no conjunctival scarring had some degree of corneal vascularisation. The corneal vascularisation became increasingly more extensive as the degree of scarring increased. That for those with a conjunctival scarring of three all had more than one millimetre of vascularisation and 40 per cent more than two millimetre vascularisation.

It appears then that conjunctival scarring is slower to appear than corneal vascularisation and that the neovascularisation of the cornea

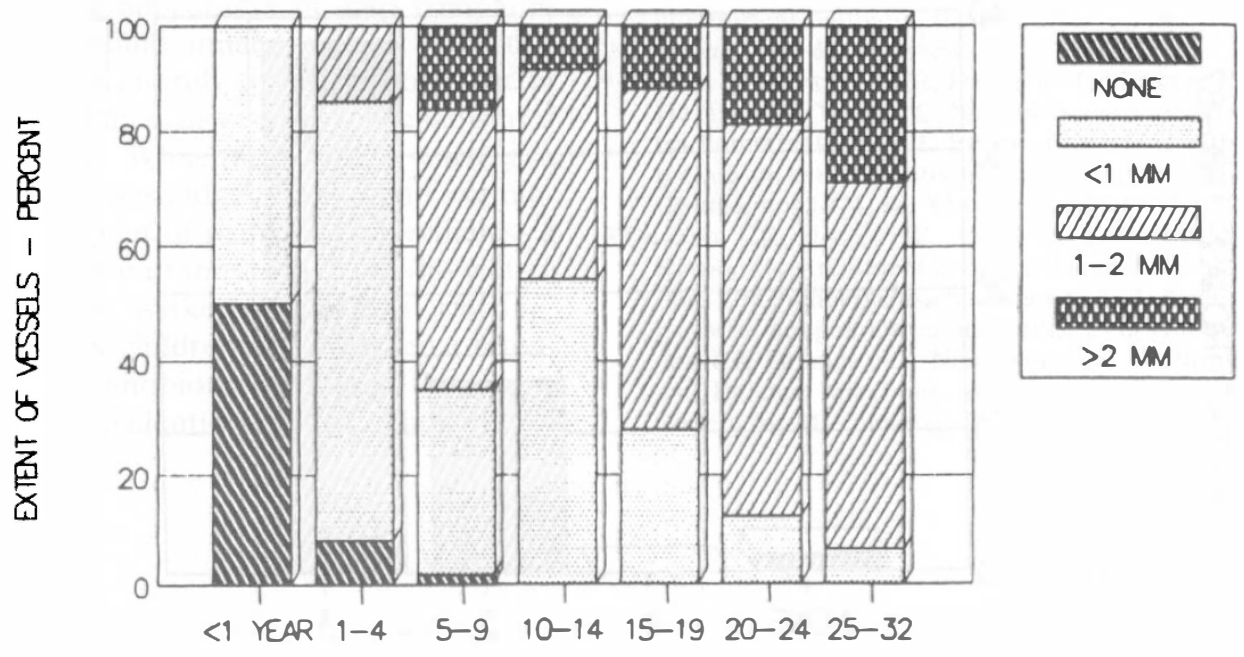

AGE AT NITIAL EXAMINATION 1969-72

Fig. 3. Trachomatous pannus 1969-1972. Corneal vascularisation with age. 


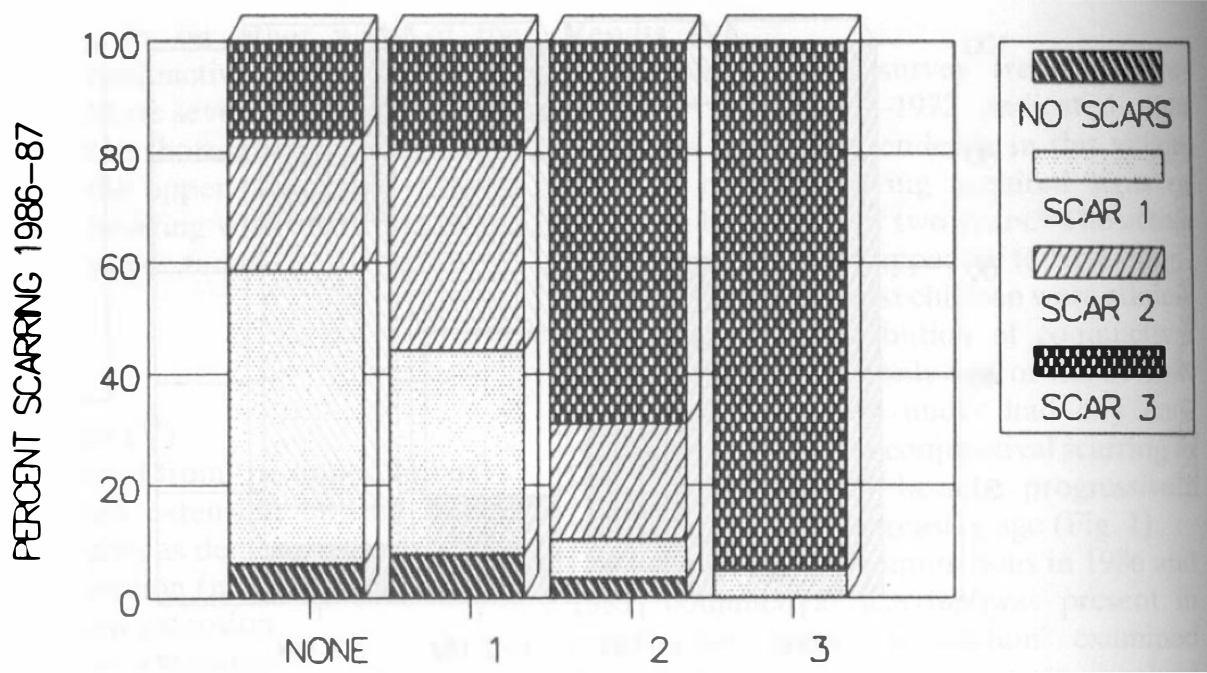

CONJUNCTVAL SCARRING 1969-1972

Fig. 4. Trachoma in Tusisia. Progression of scarring 1969 to 1987.

is a more sensitive index of conjunctival inflammation in inflammatory trachoma.

Conversely, the length of the neovascularisation is a moderately sensitive means to identify individuals at risk of developing more severe scarring in the future (Fig. 5). While only 10 per cent of those children who initially presented without pannus went on to develop severe conjunctival scarring, almost 70 per cent of those with more than two millimetre of vessel extension developed severe conjunctival scarring with lid deformity.

\section{Discussion}

Eye infections with both chlamydial infection and bacterial pathogens are highly prevalent in this community. ${ }^{8.10,11}$ Moreover, corneal infiltrates at the limbus, which are the precursors of vascular pannus, are clearly associated

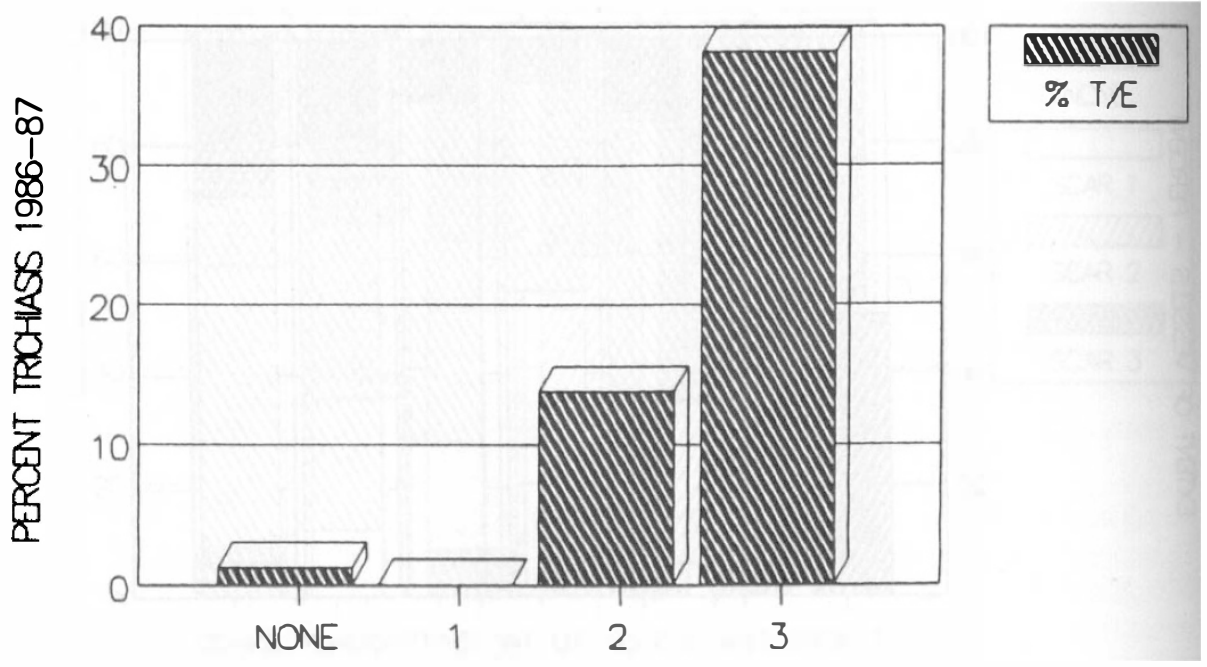

CONJUNCTIVAL SCARRING 1969-1972

Fig. 5. Trachoma in Tunisia. Conjunctival scarring and late trichiasis. 
with both chlamydial infection and with bacterial pathogens, particularly Haemophilus species, when chlamydial infection could not be confirmed. ${ }^{12}$ Thus pannus formation is due not only to chlamydial infection, but probably also accelerated or enhanced by the associated bacterial conjunctivitis. It is probable that the corneal infiltrates in trachoma are associated with the release of angiogenic factors which induce the superficial corneal vascularisation.

The mechanism for conjunctival scar formation, however, appears to be quite different. The appearance of Herbert's pits is caused primarily by dissolution of connective tissue in the scleral overhang of the limbus. The depressed pits thus left at the limbus may partially fill in with epithelium and there is some degree of new collagen formation (scars) in their base.

By analogy, then, it is quite likely that the follicles in the other parts of the conjunctiva are also capable of dissolving the local connective tissue of the conjunctiva. It has long been stated in trachoma that the conjunctival scarring occurs from the 'necrosis' of follicles and, indeed, small star-shaped scars do appear at the site of large follicles. It is probable, however, that there are other mechanisms of conjunctival scar formation which are often quite extensive and quite deep. The relatively late appearance of scars compared to pannus would indeed suggest that this mechanism is entirely different from that of pannus formation or even the mechanism of Herbert's pits. While the extent of scarring in these studies was judged by slitlamp examination, the extent of pannus is nevertheless a useful predictor of those children who will go on to develop marked conjunctival scarring. Because such children should receive intensive systemic antibiotic treatment, this sign is a useful way to identify children at high risk of developing potentially blinding complications of the disease.

\section{References}

${ }^{1}$ Duke-Elder WS: Inflammations of the conjunctiva and associated inflammations of the cornea. Specific types of keratoconjunctivitis-The TRIC viruses. In: System of Ophthalmology, Vol. VIII, Diseases of the Outer Eye, Part 1. London: Henry Kimpton, 1965: 254-303.

2 World Health Organization. Expert Committee on Trachoma. Third Report, 1962, Techn Rep Ser No 234.

${ }^{3}$ Thygeson P: Corneal changes in TRIC agent infections. Am J Ophthalmol 1967, 63: 1278-82.

${ }^{4}$ Moulder JW: Order II Chlamydiales. Storz and Page 1971, 729-739. In Krieg NR, Holt JG, eds Bergey's Manual of Systematic Microbiology, Vol. 1. Baltimore: Williams and Wilkins Co, 1984: 729-39

5 Jones BR, Al-Hussani K, Dunlop E: Infection of the eye and genital tract by TRIC agent. III. Ocular syndromes associated with infection of the genital tract by TRIC agent. Rev du Trachome 1965, 42(1): 27-43.

- Jones BR: The prevention of blindness from trachoma. Trans ophthalmol Soc UK 1975. 95: 16-33.

${ }^{7}$ Dawson CR et al.: Experimental inclusion conjunctivitis. III. Keratitis and other complications. Arch Ophthalmol 1967, 78: 341-49.

${ }^{8}$ Dawson CR et al.: Severe endemic trachoma in Tunisia. Br J Ophthalmol 1976, 60: 245-52.

${ }^{9}$ Dawson CR. Jones BR. Tarizzo M: Guide to th Control of Trachoma. Wld Hlth Org, Geneva $1981,50 \mathrm{pl}$

${ }^{10}$ Whitcher JP, Dawson CR, Messadi M, Daghfous $)^{\prime}$ ben Abdullah N. Triki F, Hoshiwara I: Severe endemic trachoma in Tunisia: Changes in ocuia: bacterial pathogens in children treated by the intermittent antibiotic regimen. Rev Int du Trachome 1974, 51(4): 44--54

1 Vastine DW, Dawson CR, Daghtous T, Messadi N: Hoshiwara 1, Yoneda C, Nataf R: Severe endemic trachoma in Tunisia. I. Effect of topicai chemotherapy on conjunctivitis and ocular bacteria. $\mathrm{Br}$; Ophthalmol 1974, 58: 833-42.

12 Dawson CR, Juster JP, Lyon C, Schachter I: Response to treatment in ocular chlamydial inie:tions (trachoma inclusion conjunctivitis): Analcgies with nongonococcal urethritis. In D Hobson? and KK Holmes eds. Nongonococcal Urethritis and Related Infections. Washington: American Society for Microbiology 1977: 135-39. 